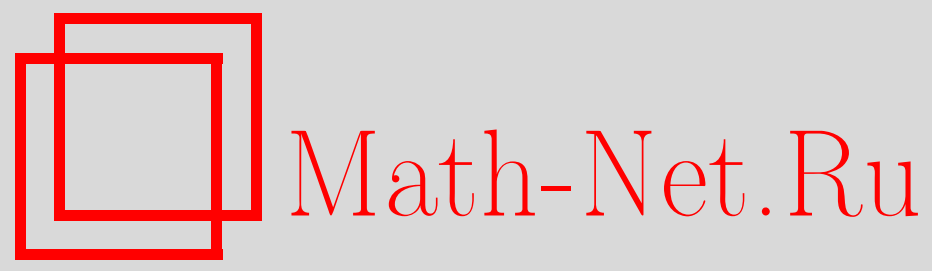

В. С. Мелентьев, Ю. М. Иванов, А. Е. Синицын, Оценка погрешности метода измерения интегральных характеристик с использованием пространственного и временного разделения мгновенных значений гармонических сигналов, Вестн. Сам. гос. техн. ун-та. Сер. Физ.-мат. науки, 2012, выпуск 3(), 199-202

DOI: https://doi.org/10.14498/vsgtu1112

Использование Общероссийского математического портала Math-Net.Ru подразумевает, что вы прочитали и согласны с пользовательским соглашением

http: //www.mathnet.ru/rus/agreement

Параметры загрузки:

IP : 3.95 .254 .165

26 апреля 2023 г., 10:14:22

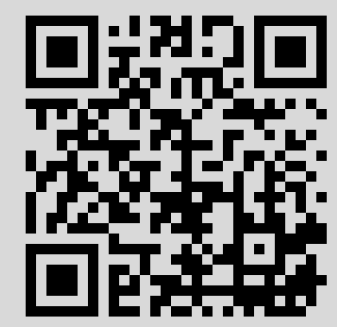


Вестн. Сам. гос. техн. ун-та. Сер. Физ.-мат. науки. 2012. № 3 (28). С. $199-202$

\title{
ОЦЕНКА ПОГРЕШНОСТИ МЕТОДА ИЗМЕРЕНИЯ ИНТЕГРАЛЬНЫХ ХАРАКТЕРИСТИК С ИСПОЛЬЗОВАНИЕМ ПРОСТРАНСТВЕННОГО И ВРЕМЕННОГО РАЗДЕЛЕНИЯ МГНОВЕННЫХ ЗНАЧЕНИЙ ГАРМОНИЧЕСКИХ СИГНАЛОВ
}

\author{
В. С. Мелентвев, Ю. М. Иванов, А.Е. Синицын \\ Самарский государственный технический университет, \\ 443100, Россия, Самара, ул. Молодогвардейская, 244. \\ E-mails: vs_mel@mail.ru, ims@samgtu.ru, AntonSinitsyn@yandex.ru
}

\begin{abstract}
Проводится анализ погрешностей метода измерения интегральных характеристик по мгновенным значениям гармонических сигналов, разделённых как в пространстве, так и во времени, исполъзующего характерные точки. Полученнье результаты позволяют выбирать оптимальные параметры измерительного процесса для обеспечения наименъшей погрешности.
\end{abstract}

Ключевые слова: интегральные характеристики, гармонические сигналь, мгновенные значения, погрешность.

Определение параметров периодических процессов находит широкое применение при контроле и испытаниях электромеханических систем, электротехнического оборудования, в системах автоматизированного управления.

В большинстве случаев периодические процессы различной физической природы преобразуются в электрические сигналы, удобные для измерения и обработки.

Особенно следует отметить гармонические сигналы, которые широко используются в измерительной технике для анализа и синтеза измерительных сигналов.

В настоящее время успешно развивается направление, связанное с разработкой методов и средств измерения интегральных характеристик по отдельным мгновенным значениям входных гармонических сигналов и дополнительных сигналов, сдвинутых относительно входных по фазе [1].

В [2] предложен метод определения интегральных характеристик гармонических сигналов (ИХГС), использующий как пространственное, так и временное разделение мгновенных значений сигналов, и отличающийся от известных использованием характерных точек (переход сигнала через ноль).

В соответствии с данным методом в момент перехода входного сигнала напряжения через ноль одновременно измеряют первое мгновенное значение дополнительного напряжения, сдвинутого по фазе относительно входного на угол $\Delta \alpha$, и первые мгновенные значения входного сигнала тока и сдвинутого относительно него по фазе на угол $\Delta \alpha$ дополнительного сигнала тока; через интервал времени $\Delta t$ одновременно измеряют вторые мгновенные значения входного и дополнительного сигналов напряжения и определяют ИХГС по измеренным значениям. Временные диаграммы, поясняющие метод, представлены на рисунке.

Владимир Сергеевич Мелентъев (д.т.н., доц.), зав. кафедрой, каф. информационно-измерительной техники. Юрий Михайлович Иванов (к.т.н.), младший научный сотрудник, каф. информационно-измерительной техники. Антон Евгенъевич Синицын, аспирант, каф. информационно-измерительной техники. 


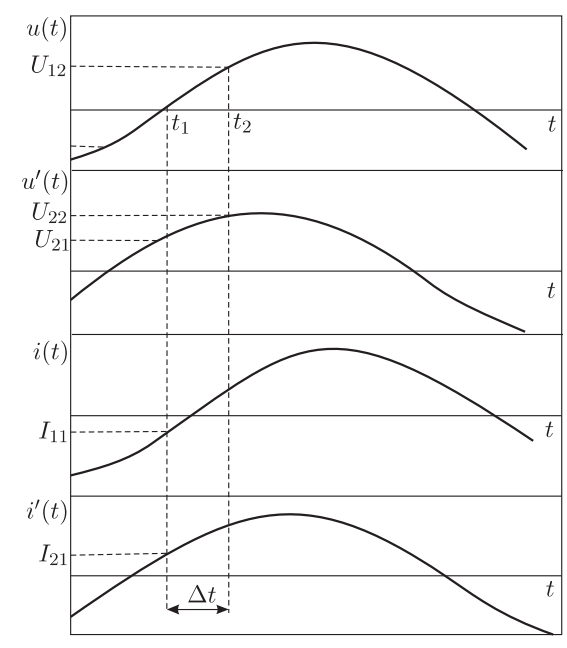

Временные диаграммы, поясняющие метод
Если сигналы напряжения и тока в исследуемой цепи имеют гармонические модели, то входные и дополнительные сигналы напряжения и тока имеют такой вид:

$$
\begin{aligned}
& u(t)=U_{m} \sin \omega t \\
& i(t)=I_{m} \sin (\omega t+\varphi) ; \\
& u^{\prime}(t)=U_{m} \sin (\omega t+\Delta \alpha) ; \\
& i^{\prime}(t)=I_{m} \sin (\omega t+\varphi+\Delta \alpha),
\end{aligned}
$$

где $U_{m}, I_{m}$ - амплитудные значения сигналов напряжения и тока; $\omega-$ угловая частота входного сигнала; $\varphi$ - угол сдвига фаз между входными сигналами напряжения и тока.

Согласно предлагаемому методу, мгновенные значения сигналов напряжения и тока следующие:

$$
\begin{gathered}
U_{21}=U_{m} \sin \Delta \alpha ; \quad U_{12}=U_{m} \sin \omega \Delta t ; \quad U_{22}=U_{m} \sin (\Delta \alpha+\omega \Delta t) ; \\
I_{11}=I_{m} \sin \varphi ; \quad I_{21}=I_{m} \sin (\varphi+\Delta \alpha) .
\end{gathered}
$$

В соответствии с (1) ИХГС задаются следующими выражениями:

- среднеквадратическими значениями (CK3) напряжения и тока:

$$
\begin{aligned}
& U_{\mathrm{CK} 3}=\left|U_{12}\right|\left(2\left[1-\left(\frac{U_{21}^{2}-U_{12}^{2}+U_{22}^{2}}{2 U_{22} U_{21}}\right)^{2}\right]\right)^{-1 / 2} ; \\
& I_{\mathrm{CK} 3}=\left(\frac { 1 } { 2 } \left[I_{11}^{2}+\frac{\left(I_{21}-I_{11} \frac{U_{22}^{2}-U_{21}^{2}+U_{12}^{2}}{2 U_{22} U_{12}}\right)^{2}}{\left.\left.\sqrt{1-\left(\frac{U_{22}^{2}-U_{21}^{2}+U_{12}^{2}}{2 U_{22} U_{12}}\right)^{2}}\right]\right)^{1 / 2} ;}\right.\right.
\end{aligned}
$$

- активной $(\mathrm{AM})$ и реактивной $(\mathrm{PM})$ мощностями:

$$
\begin{gathered}
P=\frac{\left(I_{21}-I_{11} \frac{U_{22}^{2}-U_{21}^{2}+U_{12}^{2}}{2 U_{22} U_{12}}\right)\left|U_{12}\right|}{2 \sqrt{\left[1-\left(\frac{U_{22}^{2}-U_{21}^{2}+U_{12}^{2}}{2 U_{22} U_{12}}\right)^{2}\right]\left[1-\left(\frac{U_{21}^{2}-U_{12}^{2}+U_{22}^{2}}{2 U_{22} U_{21}}\right)^{2}\right]}} ; \\
Q=\left|U_{12}\right| I_{11}\left(2 \sqrt{1-\left(\frac{U_{21}^{2}-U_{12}^{2}+U_{22}^{2}}{2 U_{22} U_{21}}\right)^{2}}\right)^{-1} .
\end{gathered}
$$

Рассматриваемый метод предназначен для определения интегральных характеристик сигналов с гармоническими моделями. При наличии в сигналах высших гармоник неизбежно возникает погрешность.

Проведём оценку методической погрешности, обусловленной отклонением реального сигнала от гармонической модели. Для этого используем предложенную в [1] методику оценки погрешности результата измерения интегральной характеристики как функции, аргументы которой заданы приближенно с погрешностью, соответствующей отклонению модели от реального сигнала. Как известно, погрешность вычисления значения какой-либо функции, аргументы которой заданы приближенно, может быть оценена с помощью дифференциала этой функции. Погрешность 
функции есть не что иное, как возможное приращение функции, которое она получит, если её аргументам дать приращения, равные их погрешностям.

Пусть предельные абсолютные погрешности аргументов соответствуют наибольшему отклонению моделей от реальных сигналов. В этом случае предельные значения абсолютных погрешностей определения интегральных характеристик сигналов в соответствии с (2)-(5) определяются так:

$$
\begin{gathered}
\Delta U_{\mathrm{CK} 3}=\left[\left|\left(U_{\mathrm{CK} 3}\right)_{U_{21}}^{\prime}\right|+\left|\left(U_{\mathrm{CK} 3}\right)_{U_{12}}^{\prime}\right|+\left|\left(U_{\mathrm{CK} 3}\right)_{U_{22}}^{\prime}\right|\right] \Delta U_{\max } \\
\Delta I_{\mathrm{CK} 3}=\left[\left|\left(I_{\mathrm{CK} 3}\right)_{I_{11}}^{\prime}\right|+\left|\left(I_{\mathrm{CK} 3}\right)_{I_{21}}^{\prime}\right|\right] \Delta I_{\max }+ \\
+\left[\left|\left(I_{\mathrm{CK} 3}\right)_{U_{21}}^{\prime}\right|+\left|\left(I_{\mathrm{CK} 3}\right)_{U_{12}}^{\prime}\right|+\left|\left(I_{\mathrm{CK} 3}\right)_{U_{22}}^{\prime}\right|\right] \Delta U_{\max } ; \\
\Delta=\left[\left|(P)_{I_{11}}^{\prime}\right|+\left|(P)_{I_{21}}^{\prime}\right|\right] \Delta I_{\max }+\left[\left|(P)_{U_{21}}^{\prime}\right|+\left|(P)_{U_{12}}^{\prime}\right|+\left|(P)_{U_{22}}^{\prime}\right|\right] \Delta U_{\max } ; \\
\Delta Q=\left|(Q)_{I_{11}}^{\prime}\right| \Delta I_{\max }+\left[\left|(Q)_{U_{21}}^{\prime}\right|+\left|(Q)_{U_{12}}^{\prime}\right|+\left|(Q)_{U_{22}}^{\prime}\right|\right] \Delta U_{\max },
\end{gathered}
$$

где $\Delta U_{\max }, \Delta I_{\max }$ - предельные абсолютные погрешности аргументов, соответствующие наибольшим отклонениям моделей от реальных сигналов.

В общем случае $\Delta U_{\max }=U_{1 m} \sum_{k=2}^{\infty} h_{u k}$ и $\Delta I_{\max }=I_{1 m} \sum_{k=2}^{\infty} h_{i k}$, где $h_{u k}$ и $h_{i k}-$ коэффициенты $k$-тых гармоник напряжения и тока; $U_{1 m}$ и $I_{1 m}$ - амплитуды первых гармоник сигналов.

Используя выражения (2)-(5) и предельные значения абсолютных погрешностей (6)-(9), можно определить относительные погрешности определения CK3 напряжения и тока и приведенные погрешности определения АМ и РМ:

$$
\begin{aligned}
& \delta_{U_{\mathrm{CК} 3}}=\frac{\sum_{k=2}^{\infty} h_{u k}[|\cos (\Delta \alpha+\omega \Delta t)|(|\cos \omega \Delta t|+|\cos \Delta \alpha|)+|\cos \omega \Delta t \cos \Delta \alpha|]}{\sqrt{1+\sum_{k=2}^{\infty} h_{u k}^{2}}|\sin \omega \Delta t \sin \Delta \alpha \sin (\Delta \alpha+\omega \Delta t)|} ; \\
& \delta_{I_{\mathrm{CK} 3}}=\frac{\sum_{k=2}^{\infty} h_{i k}[|\cos \varphi|+|\cos (\varphi+\Delta \alpha)|]}{\sqrt{1+\sum_{k=2}^{\infty} h_{i k}^{2}}|\sin \Delta \alpha|}+ \\
& +\frac{\sum_{k=2}^{\infty} h_{u k}[|\cos \varphi \cos (\varphi+\Delta \alpha)|]}{\sqrt{1+\sum_{k=2}^{\infty} h_{u k}^{2}}|\sin \omega \Delta t \sin \Delta \alpha \sin (\Delta \alpha+\omega \Delta t)|} \times \\
& \times[1+|\cos (\Delta \alpha+\omega \Delta t)|+|\cos \omega \Delta t|] ; \\
& \gamma_{P}=\frac{1}{\sqrt{1+\sum_{k=2}^{\infty} h_{u k}^{2}} \sqrt{1+\sum_{k=2}^{\infty} h_{i k}^{2}}|\sin \Delta \alpha|}\left(\sum_{k=2}^{\infty} h_{i k}(|\cos \Delta \alpha|+1)+\right. \\
& +\frac{\sum_{k=2}^{\infty} h_{u k}}{|\sin \omega \Delta t \sin (\Delta \alpha+\omega \Delta t)|}\{|\cos \omega \Delta t \cos \varphi \cos (\Delta \alpha+\omega \Delta t)+\cos (\Delta \alpha+\varphi)|+ \\
& +|\cos \varphi \cos \Delta \alpha+\cos (\Delta \alpha+\varphi)|[|\cos (\Delta \alpha+\omega \Delta t)|+|\cos \omega \Delta t|]\}) \\
& \gamma_{Q}=\frac{1}{\sqrt{1+\sum_{k=2}^{\infty} h_{u k}^{2}} \sqrt{1+\sum_{k=2}^{\infty} h_{i k}^{2}}}\left(\sum_{k=2}^{\infty} h_{i k}+\frac{\sum_{k=2}^{\infty} h_{u k}|\sin \varphi|}{|\sin \Delta \alpha \sin \omega \Delta t \sin (\Delta \alpha+\omega \Delta t)|} \times\right. \\
& \times\{|\cos (\Delta \alpha+\omega \Delta t) \cos \Delta \alpha|+|\cos \omega \Delta t|[|\cos \Delta \alpha|+|\cos (\Delta \alpha+\omega \Delta t)|]\}) .
\end{aligned}
$$


Анализ выражений (10)-(13) показывает, что относительные погрешности определения СКЗ напряжения и тока и приведенные погрешности определения АМ и РМ зависят от гармонического состава сигналов, угла сдвига фаз между входными и дополнительными сигналами $\Delta \alpha$ и интервала времени $\Delta t$. Кроме того, погрешности $\delta_{I_{\mathrm{CK} 3}}, \gamma_{P}$ и $\gamma_{Q}$ зависят от угла сдвига фаз между напряжением и током $\varphi$.

Полученные результаты позволяют выбирать области использования метода в зависимости от спектра сигналов и требований по точности измерения, а также подбирать оптимальные параметры измерительного процесса для обеспечения наименьшей погрешности.

Работа выполнена при поддержке РФФИ (проект № 11-08-00039-а).

\title{
БИБЛИОГРАФИЧЕСКИЙ СПИСОК
}

1. Мелентвев B.С., Батищев В.И. Аппроксимационные методы и системы измерения и контроля параметров периодических сигналов. М.: Физматлит, 2011. 240 с. [Melentiev V.S., Batishev V.I. Approximation methods and systems for measuring and parameters monitoring of periodic signals. Moscow: Fizmatlit, 2011. 240 pp.]

2. Мелентвев В.С., Леонович Г. И., Кожевникова Е.Г. Метод измерения интегральных характеристик по мгновенным значениям гармонических сигналов, разделённым во времени и пространстве / В сб.: Компьютерные технологии в науке, практике и образовании: Тр. 9 Всерос. межвуз. науч.-практ. конф. Самара: СамГТУ, 2010. С. 4244. [Melentiev V.S., Leonovich G. I., Kozhevnikova E. G. Integral characteristics measuring method by instantaneous values of harmonic signals separated in time and space/ In: Computer technology in the science, practice and education. Samara: SamGTU, 2010. Pp. 4244].

Поступила в редакцию $12 / \mathrm{V} / 2012$;

в окончательном варианте - 23/VIII/2012.

MSC: $42 \mathrm{~A} 10$

\section{ERROR ESTIMATION OF METHOD OF INTEGRAL CHARACTERISTICS MEASUREMENT USING SPATIAL AND TIME DIVISION OF HARMONIC SIGNALS INSTANT VALUES}

\author{
V.S. Melentiev, Yu. M. Ivanov, A.E. Sinitsyn
}

Samara State Technical University,

244, Molodogvardeyskaya st., Samara, 443100, Russia.

E-mails: vs_mel@mail.ru, ims@samgtu.ru, AntonSinitsyn@yandex.ru

The analysis of errors of a method of integral characteristics measurement on instant values of harmonic signals divided both in space and in time, using characteristic points, is carried out. The received results allow choosing optimum parameters of measuring process for providing the least error.

Key words: integral characteristics, harmonic signals, instant values, error.

Original article submitted $12 / \mathrm{V} / 2012$; revision submitted $23 / \mathrm{VIII} / 2012$.

Vladimir S. Melentiev (Dr. Sci. (Techn.)), Head of Dept., Dept. of Information and Measuring Technics. Yuriy M. Ivanov (Ph. D. (Techn.)), Associate Researcher, Dept. of Information and Measuring Technics. Anton E. Sinitsyn, Postgraduate Student, Dept. of Information and Measuring Technics. 